Supporting Information

\title{
A self-assembled FRET nanoprobe with metal-organic framework as a scaffold for ratiometric detection of hypochlorous acid
}

Hongliang Tan*, Xiayi Wu, Yuhao Weng, Yajie Lu, Zhen-Zhong Huang

Key Laboratory of Chemical Biology of Jiangxi Province, College of Chemistry and Chemical Engineering, Jiangxi Normal University, Nanchang, 330022, P. R. China.

*Corresponding author: E-mail: hltan@jxnu.edu.cn 


\section{Table of Contents}

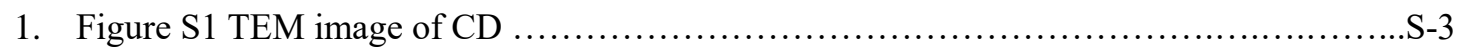

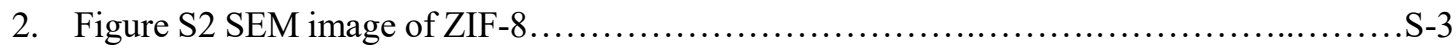

3. Figure S3 Powder XRD patterns of pure ZIF-8 and CD/CCM@ZIF-8..................................S-4

4. Figure S4 Absorption spectra of CD, CCM, ZIF-8 and CD/CCM@ZIF-8 ...............................S-4

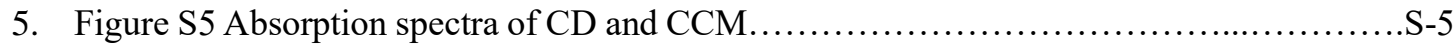

6. Figure S6 Release profiles of CD/CCM@ZIF-8 ……………................................................

7. Figure S7 Emission spectra of the solution of free CCM after adding ZIF-8 and the solution of ZIF-8 adding free CCM.

8. Figure S8 Emission spectra of free CCM at different concentrations and absorption spectra of

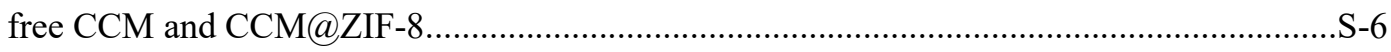

9. Figure S9. Emission spectra of free CCM and CCM@ZIF-8 in the presence of CD...........S-7

10. Figure S10 Absorption spectra of free CCM in the presence of CD...................... -7

11. Figure S11 Emission spectra of free CD in the presence of CCM......................... 8

12. Figure $\mathrm{S} 12$ Excitation and emission spectra and decay curves of free $\mathrm{CD}$ and the mixture of free $\mathrm{CD}$ and $\mathrm{CCM}$.

13. Figure S13 Emission spectra of CD/CCM@ZIF-8 with different concentration of CCM and the plot of FRET efficiency with different concentration of CCM................................

14. Figure $\mathrm{S} 14$ Effects of $\mathrm{HClO}$ on the fluorescence of free $\mathrm{CD}$ and free $\mathrm{CCM} \ldots \ldots \ldots \ldots \ldots . . . . . . .5$

15. Figure S15 Emission spectra of CCM@ZIF-8 in the presence of HClO.....................S-10

16. Figure S16 Time-dependent $F_{410} / F_{585}$ of CD/CCM@ZIF-8 in the presence of HClO........S-10

17. Figure $\mathrm{S} 17$ Effects of interferential substances on the detection of $\mathrm{HClO}$................................ -11

18. Figure S18 Fluorescent stability of CD/CCM@ZIF-8 in PBS buffer..................................S-11

19. Figure S19 Structural stability of CD/CCM@ZIF-8 in serum sample......................S-12

20. Table S1 Comparison of various fluorescent methods for $\mathrm{HClO}$ detection.................. S-12

21. Table S2 Determination of $\mathrm{HClO}$ in spiked serum samples................................ 13

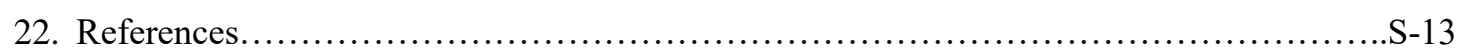




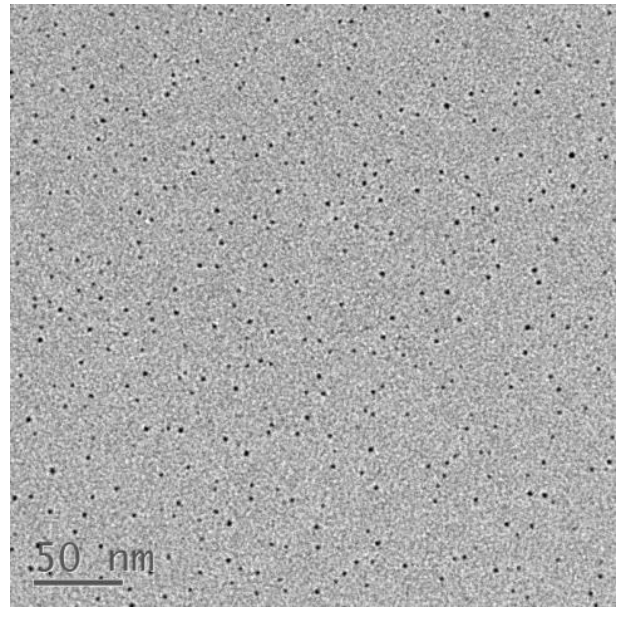

Figure S1. TEM image of the CD synthesized by using salicylic acid as a carbon source under hydrothermal condition.

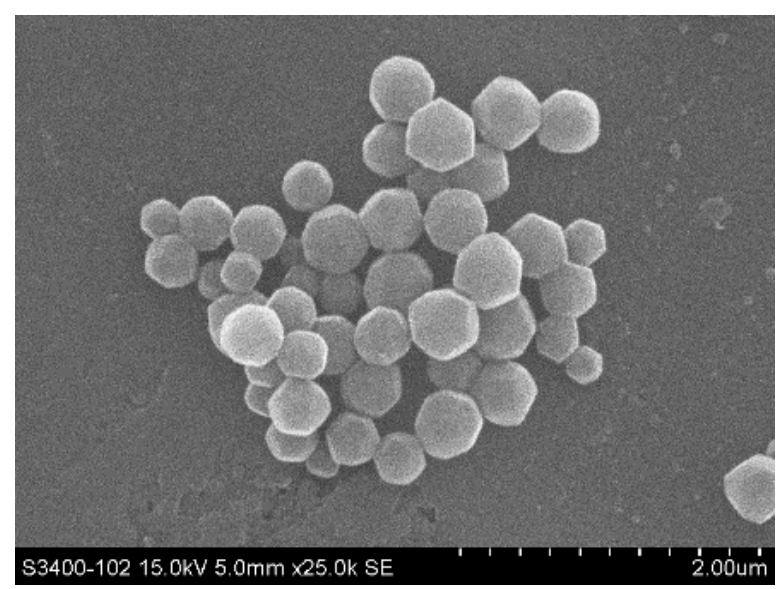

Figure S2. SEM image of pure ZIF-8. 


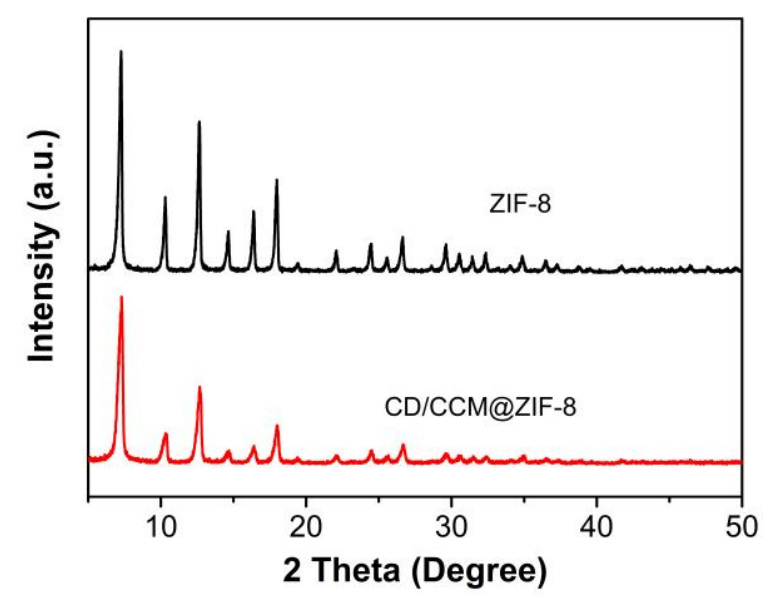

Figure S3. Powder XRD patterns of pure ZIF-8 and CD/CCM@ZIF-8.

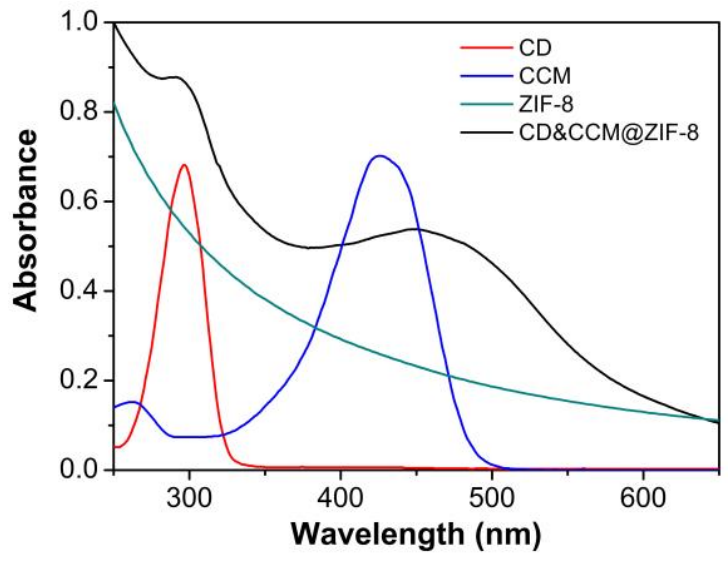

Figure S4. Absorption spectra of CD, CCM, ZIF-8 and CD/CCM@ZIF-8. 

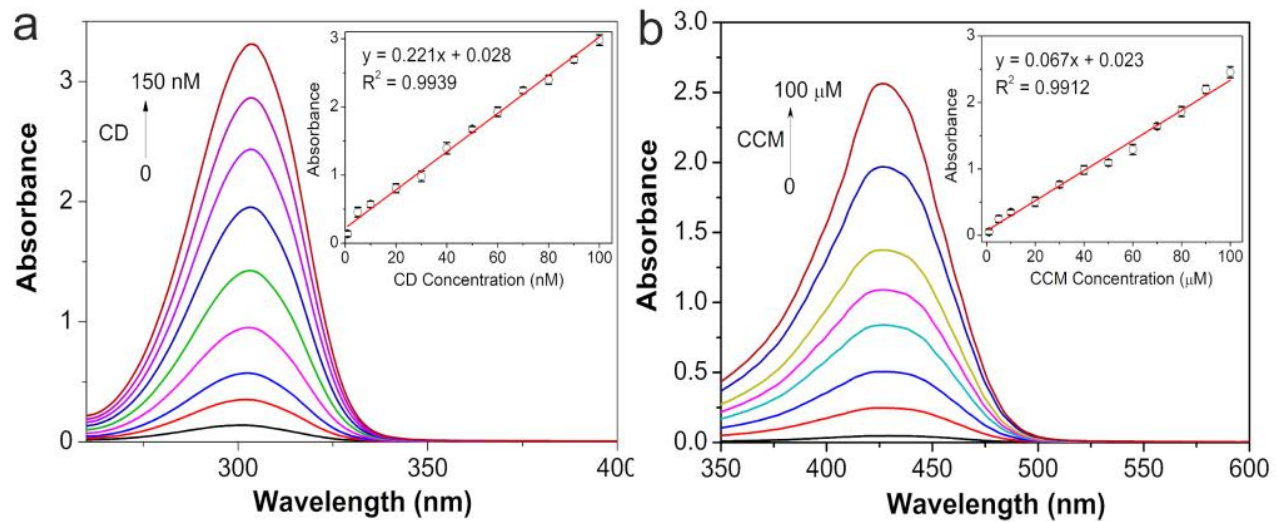

Figure S5. Absorption spectra of CD (a) and CCM (b). Insets are their corresponding calibration curves versus $\mathrm{CD}$ or $\mathrm{CCM}$ concentrations.

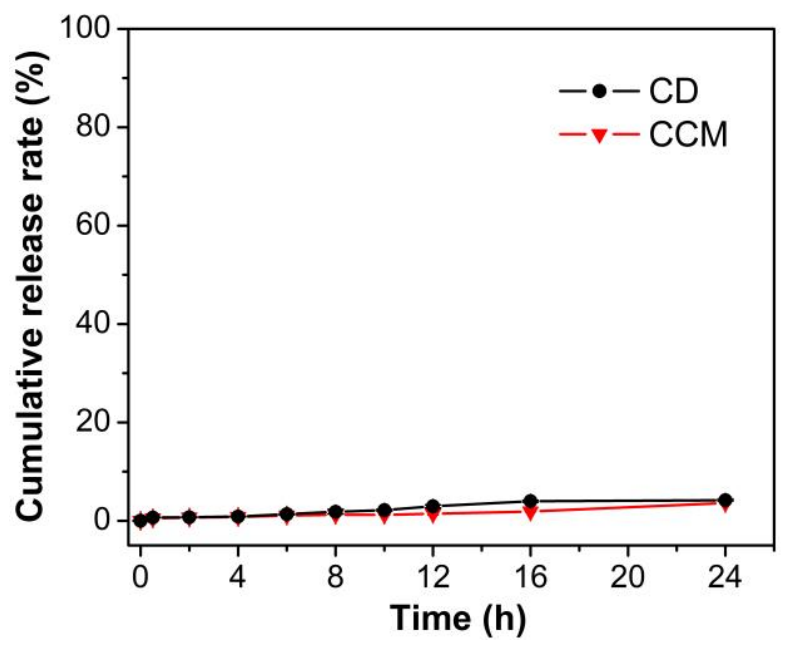

Figure S6. Release profiles of CD and CCM from CD/CCM@ZIF-8. 

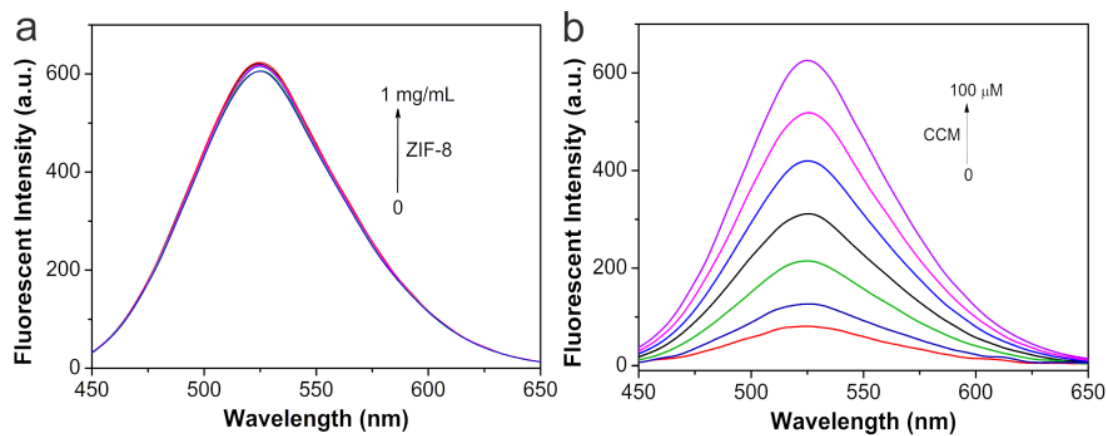

Figure S7. Emission spectra of (a) the solution of free CCM after adding ZIF-8 and (b) the solution of ZIF-8 adding free CCM.
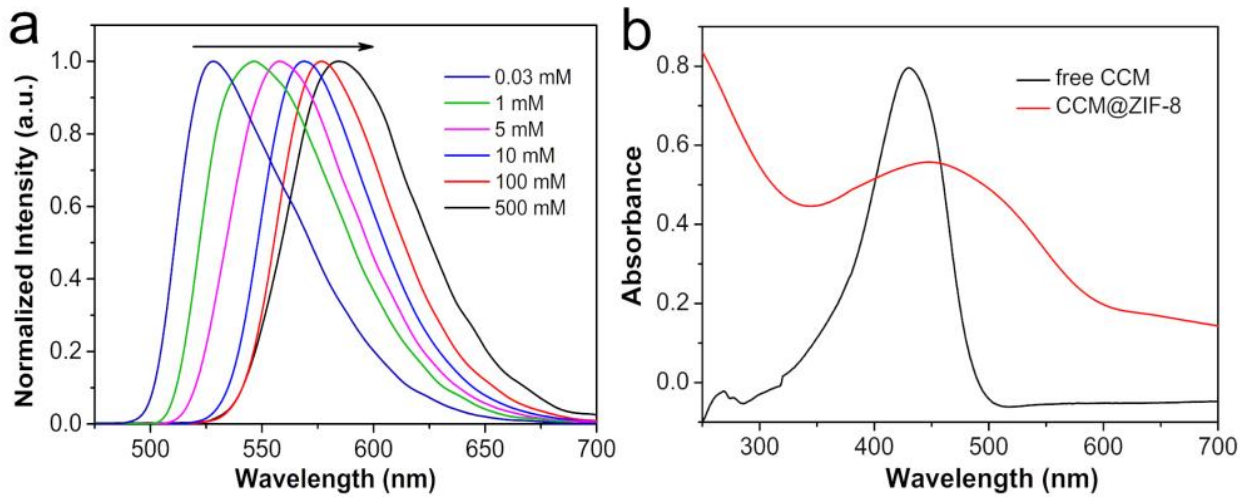

Figure S8. (a) Emission spectra of free CCM at different concentrations (from $30 \mu \mathrm{M}$ to $500 \mathrm{mM}$ ). (b) Absorption spectra of free CCM and CCM@ZIF-8. 

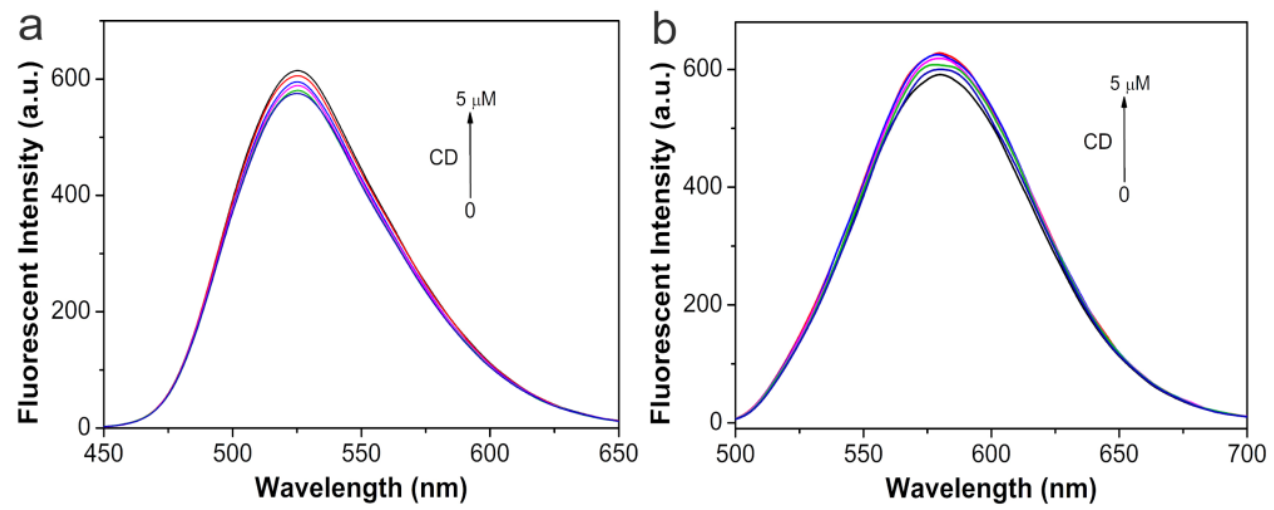

Figure S9. Emission spectra of free CCM (a) and CCM@ZIF-8 (b) in the presence of CD.

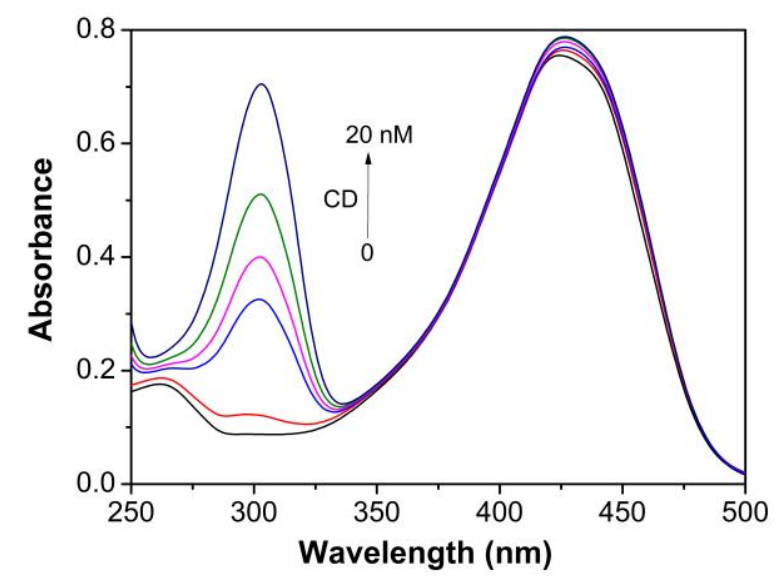

Figure S10. Absorption spectra of free CCM in the presence of CD. 


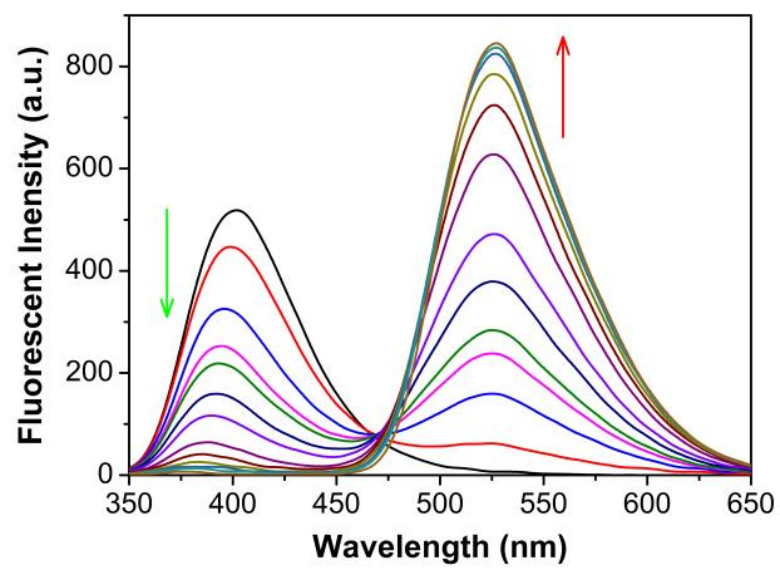

Figure S11. Emission spectra of free CD after adding CCM with concentration from 0 to $90 \mu \mathrm{M}$.
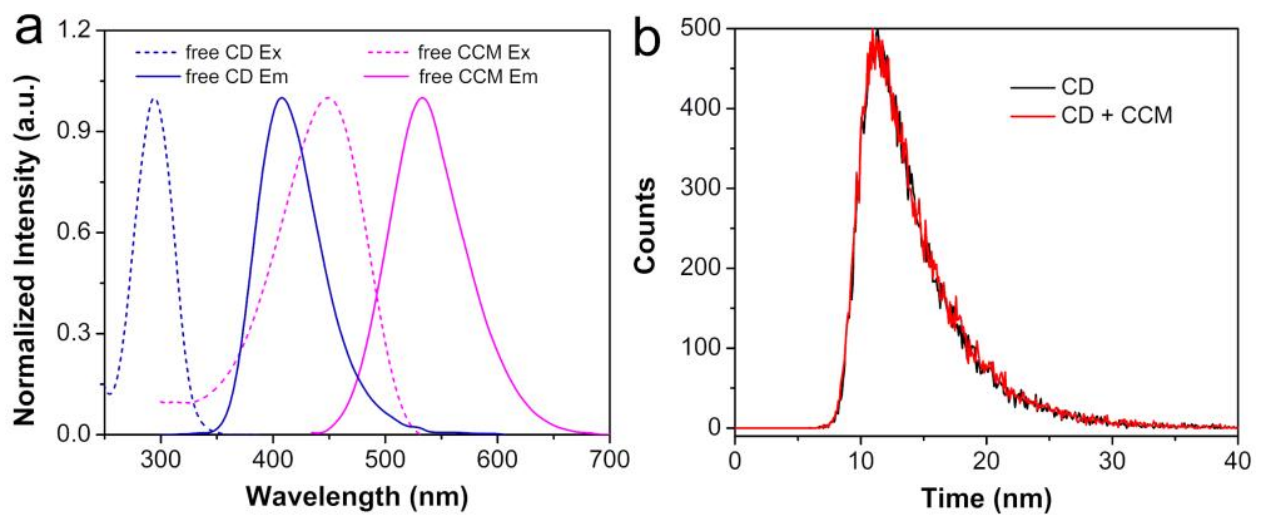

Figure S12. Excitation and emission spectra (a) and decay curves (b) of free CD and the mixture of free $\mathrm{CD}$ and $\mathrm{CCM}$. 

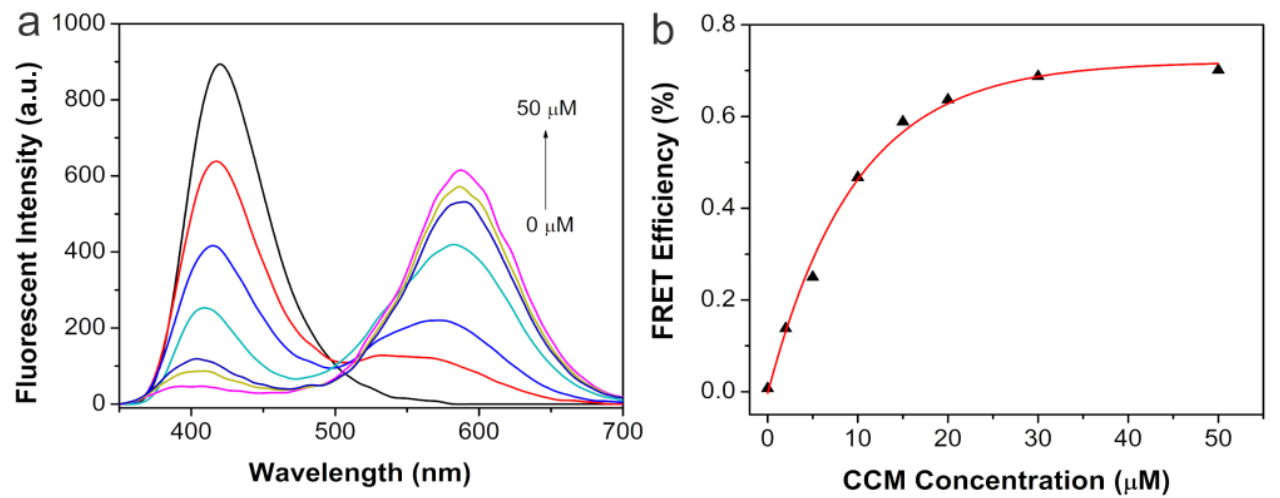

Figure S13. (a) Emission spectra of CD/CCM@ZIF-8 with different concentration of CCM (from bottom to up: $0,4.75,9.12,14.87,28.5,30,50 \mu \mathrm{M}$ ), CD concentration was at $25 \mathrm{nM}$; (b) Plot of FRET efficiency with different concentration of CCM.
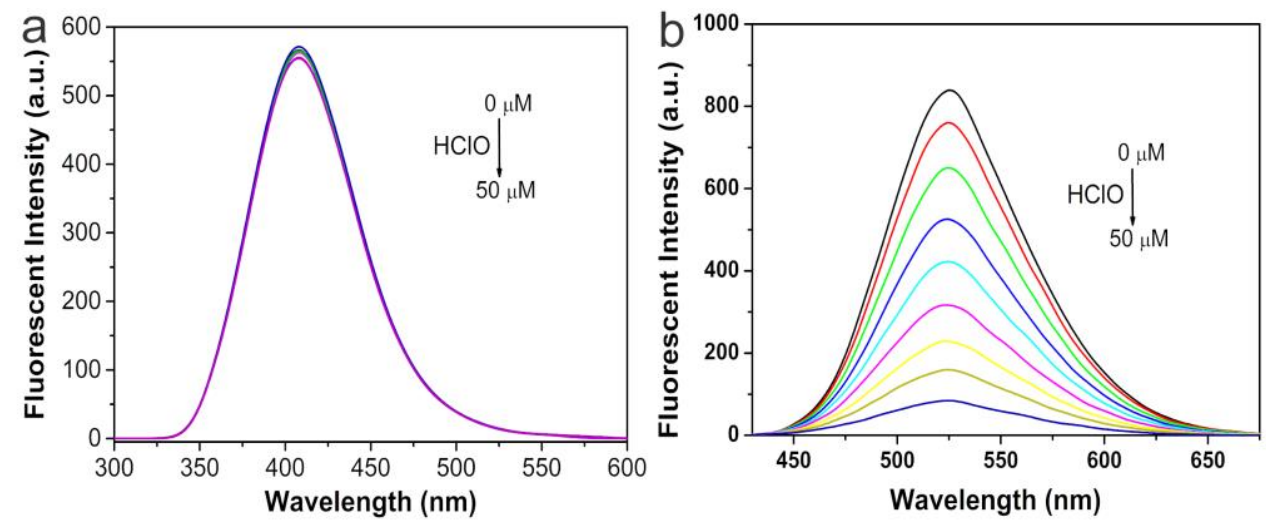

Figure S14. Effects of $\mathrm{HClO}$ on the fluorescence of free $\mathrm{CD}$ (a) and free CCM (b). 


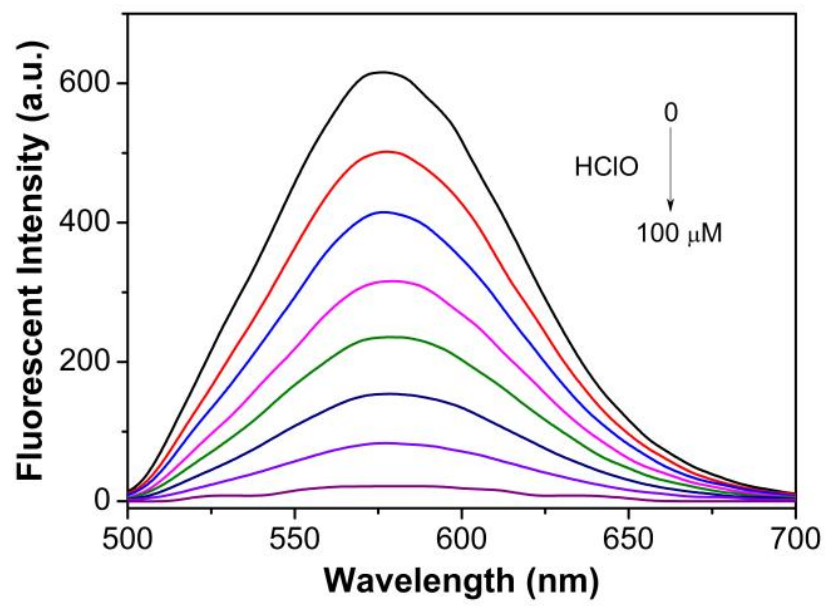

Figure S15. Emission spectra of CCM@ZIF-8 in the presence of HClO.

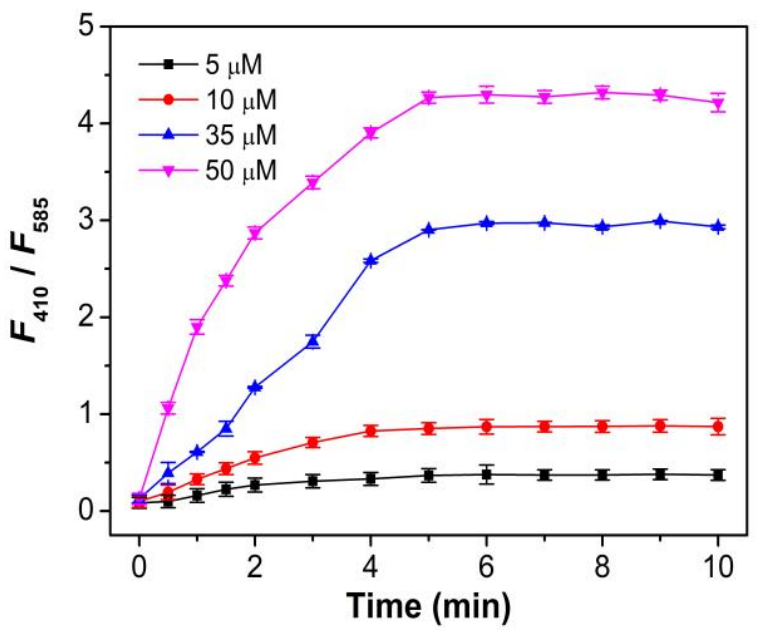

Figure S16. Time-dependent changes of $F_{410} / F_{585}$ value of CD/CCM@ZIF-8 in the presence of $\mathrm{HClO}$ with different concentrations $(5,10,35$, and $50 \mu \mathrm{M})$. 


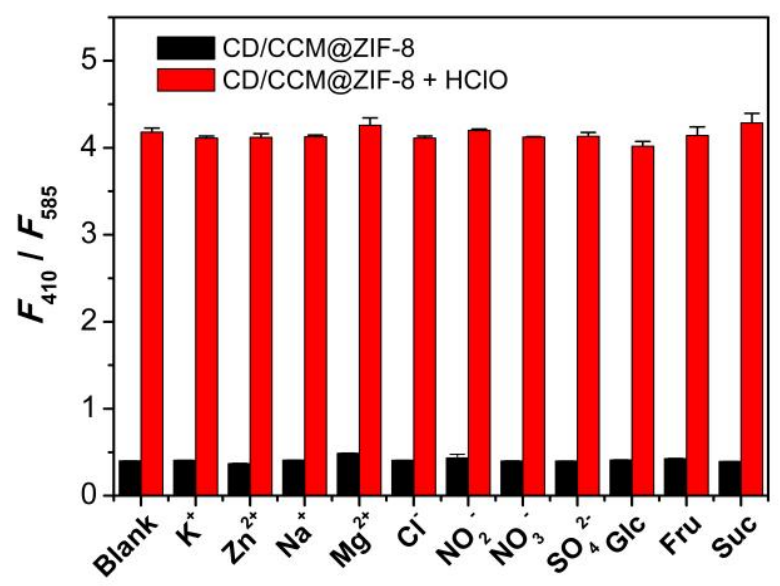

Figure S17. The changes of $F_{410} / F_{585}$ value of CD/CCM@ZIF-8 in the presence of $\mathrm{HClO}(50 \mu \mathrm{M})$ and interferential substances (each $200 \mu \mathrm{M}$ ). Black bars represent the addition of interferential substance $(200 \mu \mathrm{M})$; Red bars represent the addition of interferential substance $(200 \mu \mathrm{M})$ and $\mathrm{HClO}$ $(50 \mu \mathrm{M})$ together.
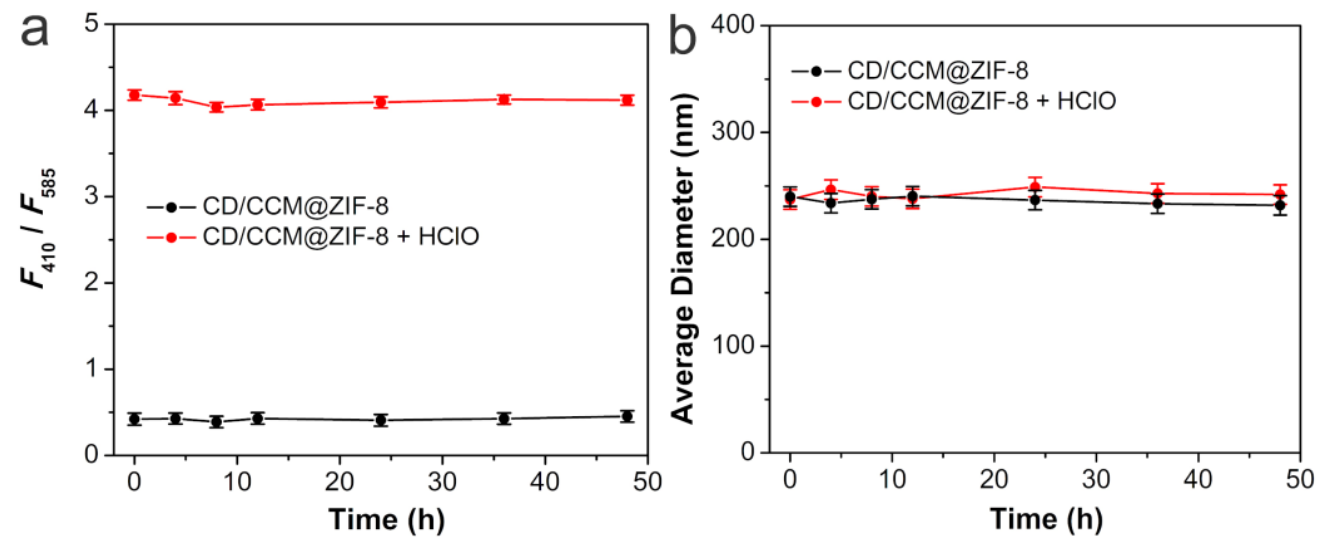

Figure S18. The changes of $F_{410} / F_{585}$ value (a) and hydrodynamic diameters (b) of CD/CCM@ZIF8 and CD/CCM@ZIF-8 + $50 \mu \mathrm{M} \mathrm{HClO}$ after keeping in PBS buffer $(50 \mathrm{mM}, \mathrm{pH}=7.4)$ for different times at room temperature. 


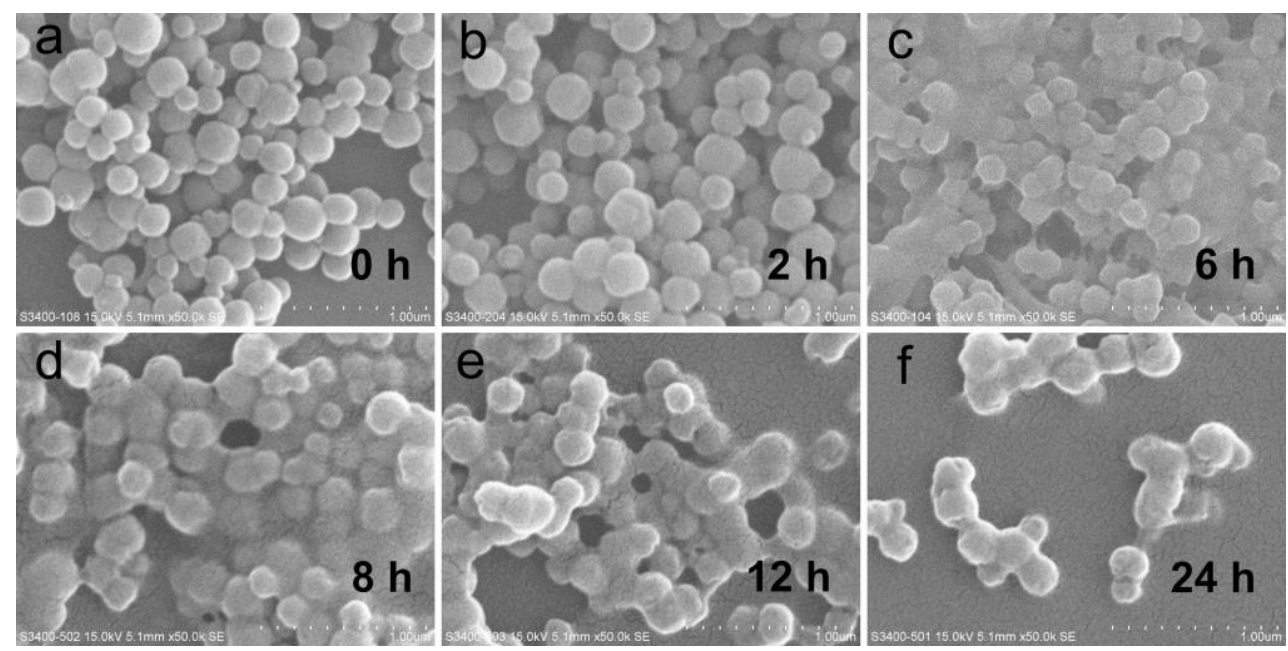

Figure S19. SEM images of CD/CCM@ZIF-8 after storing in serum sample for $0 \mathrm{~h}$ (a), 2 h (b), $6 \mathrm{~h}$ (c), $8 \mathrm{~h} \mathrm{(d),} 12 \mathrm{~h}$ (e), and $24 \mathrm{~h}$ (f).

Table S1. Comparison of various fluorescent methods for $\mathrm{HClO}$ detection.

\begin{tabular}{llccc}
\hline \multicolumn{1}{c}{ Signal modes } & \multicolumn{1}{c}{ Materials } & $\begin{array}{c}\text { Linear range } \\
(\mu \mathrm{M})\end{array}$ & $\begin{array}{c}\text { Detection limit } \\
(\mathrm{nM})\end{array}$ & Refs. \\
\hline Single wavelength & Europium complex & $0.1-4$ & 90 & 1 \\
Single wavelength & BODIPY derivative & $0-6$ & 17.7 & 2 \\
Single wavelength & Synthesized FBS & $0-1$ & 200 & 3 \\
Single wavelength & CCM & $0-5$ & 65 & 4 \\
Single wavelength & Copper nanocluster & $1-30$ & 100 & 5 \\
Ratiometric & Cy7/RhB@SiO 2 & $0-50$ & N.A. & 6 \\
Ratiometric & Coumarin/RhB & $0-50$ & 70 & 7 \\
Ratiometric & Polymer dots & $0-4$ & 5.1 & 8 \\
Ratiometric & CD-RhB@ZIF-8 & $15-180$ & 6700 & 9 \\
Ratiometric & CD/CCM@ZIF-8 & $0.1-50$ & 67 & This work \\
\hline
\end{tabular}

N.A. : Not Available. 
Table S2. Determination of HClO in spiked serum samples

\begin{tabular}{ccccc} 
Added $(\boldsymbol{\mu M})$ & Detected $(\boldsymbol{\mu M})$ & Recovery $(\%)$ & $\mathbf{R S D}(\mathbf{n}=\mathbf{5}, \boldsymbol{\%})$ & KIT assay $(\boldsymbol{\mu M})$ \\
\hline 0 & - & - & - & - \\
3 & 2.92 & 97.33 & 2.31 & 3.02 \\
10 & 10.38 & 103.80 & 1.87 & 10.31 \\
20 & 20.05 & 100.25 & 2.12 & 20.27 \\
\hline
\end{tabular}

\section{References}

1. Wang, Q.; Tan, C.; Cai, W., A targetable fluorescent sensor for hypochlorite based on a luminescent europium complex loaded carbon nanotube. Analyst 2012, 137, 1872-1875.

2. Wu, G.; Zeng, F.; Wu, S., A water-soluble and specific BODIPY-based fluorescent probe for hypochlorite detection and cell imaging. Anal. Methods 2013, 5, 5589-5596.

3. Xu, Q.; Lee, K.-A.; Lee, S.; Lee, K. M.; Lee, W.-J.; Yoon, J., A highly specific fluorescent probe for hypochlorous acid and its application in imaging microbe-induced $\mathrm{HOCl}$ production. J. Am. Chem. Soc. 2013, 135, 9944-9949.

4. Yue, Y.; Yin, C.; Huo, F.; Chao, J.; Zhang, Y., The application of natural drug-curcumin in the detection hypochlorous acid of real sample and its bioimaging. Sens. Actuators B-Chem. 2014, 202, 551-556.

5. Tang, Q.; Yang, T.; Huang, Y., Copper nanocluster-based fluorescent probe for hypochlorite. Microchim. Acta 2015, 182, 2337-2343.

6. Chen, G.; Song, F.; Wang, J.; Yang, Z.; Sun, S.; Fan, J.; Qiang, X.; Wang, X.; Dou, B.; Peng, X., FRET spectral unmixing: a ratiometric fluorescent nanoprobe for hypochlorite. Chem. Commun. 2012, 48, 2949-2951.

7. Shen, S.-L.; Zhao, X.; Zhang, X.-F.; Liu, X.-L.; Wang, H.; Dai, Y.-Y.; Miao, J.-Y.; Zhao, B.-X., A mitochondria-targeted ratiometric fluorescent probe for hypochlorite and its applications in bioimaging. J. Mater. Chem. B 2017, 5, 289-295.

8. Wang, H.; Zhang, P.; Hong, Y.; Zhao, B.; Yi, P.; Chen, J., Ratiometric imaging of lysosomal hypochlorous acid enabled by FRET-based polymer dots. Polym. Chem. 2017, 8, 5795-5802.

9. Ma, Y.; Xu, G.; Wei, F.; Cen, Y.; Xu, X.; Shi, M.; Cheng, X.; Chai, Y.; Sohail, M.; Hu, Q., Onepot synthesis of a magnetic, ratiometric fluorescent nanoprobe by encapsulating $\mathrm{Fe}_{3} \mathrm{O}_{4}$ magnetic nanoparticles and dual-emissive rhodamine $\mathrm{B}$ modified carbon dots in metal-organic framework for enhanced HClO sensing. ACS Appl. Mater. Interfaces 2018, 10, 20801-20805. 